\title{
Téoros
}

Revue de recherche en tourisme

\section{Tendances et défis du tourisme international}

Idées-forces d'un séminaire récent de l'Organisation mondiale du tourisme à Montréal

\section{Le Gouvernement du Québec, Ministère du Tourisme}

Volume 12, numéro 2, juillet 1993

La prévision-prospective du tourisme

URI : https://id.erudit.org/iderudit/1078023ar

DOI : https://doi.org/10.7202/1078023ar

Aller au sommaire du numéro

Éditeur(s)

Université du Québec à Montréal

ISSN

0712-8657 (imprimé)

1923-2705 (numérique)

Découvrir la revue

Citer ce compte rendu

Le Gouvernement du Québec, Ministère du Tourisme (1993). Compte rendu de

[Tendances et défis du tourisme international : idées-forces d'un séminaire

récent de l'Organisation mondiale du tourisme à Montréal]. Téoros, 12(2),

27-34. https://doi.org/10.7202/1078023ar 
Gouvernement du Québec Ministère du Tourisme

\author{
Idées-forces d'un séminaire récent de \\ I'Organisation mondiale du tourisme à Montréal
}

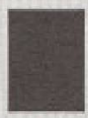

\section{Présentation}

Le Secrétariat régional des membres affiliés de IrOMT pour les Amériques instal lé a Montreal (380 rue St-Antoine, ouest, suite 3200, H2Y 3X7, (514) 288-0501), a pris l'initiative d'organiser dans cette ville un séminaire de 2 jours sur les tendances et défis du tourisme international. Cette rencontre a eu lieu les 27 et 28 mai derniers au Palais des congrès, Les pages qui suivent, habituellement réservées à la publicité du ministèredu Tourisme du Québec, ont été confiées aux responsables du séminaire de I'OMT pour rendre compte de cet événement et en faire ressortir les principales contributions. II s'agit d'une initiative des plus heureuses de la part du ministère du Tourisme du Québec puisque le présent compte-rendu de ce séminaire vient compléter de façon très pertinente la liste déja impressionnante des articles du présent numéro de Téoros, consacré à la prévisionprospective du tourisme.

\begin{abstract}
La rédaction de ces comptes-rendus est l'oeuvre de madame Isabelle Mayer, assistée de madame Odette Rivard (pour le texte sur I'OMT), étudiantes au baccalauréaten gestion et intervention touristiques de I'UOAM. Les photographies sont de monsieur Mare Roman, enseignant au Département d'études urbaines et touristiques de I'UOAM.
\end{abstract}

Avant d'aborder comme tel les exposés du séminaire, il importe de présenter succinctement I'Organisation mondiale du tourisme (OMT) et ses divers champs d'action.

\section{L'OMT: une organisation internationale dynamique}

L'Organisation mandiale du tourisme fut créée en 1975 pour succéder à l'Union internationale des organismes officiels de tourisme, fondeeen 1947. Affiliée aux Nations Unies, ayant son siège social a Madrid, I'OMT compte aujourd' hui 110 gouvernements membres de pleins droits qui ont la responsabilité du développement mondial du tourisme comme secteur economique et comme facteur de paix et de compréhension entre les nations. L'OMT reconnait aussi des membres affiliés, au nombre de 190 actuellement, représentant tous les grands intervenants de l'industrie touristique. Les rencontres des secteurs public et privéauplus haut niveau sont les moyens privilégiés pour que l'Organisation atteigne ses objectifs.

L'OMT agit de nombreuses façons pour le bénéfice du développement touristique mondial; a) elle fournit des conseils et de l'assistance aux gowvernements, à titre de coopération technique, puisqu'elle est agent d'exécution du Programme des Nations Unies pour le développement (PNUDI pour les questions touristiques; b) elle s'occupe d'éducation et de formation en tourisme et no-

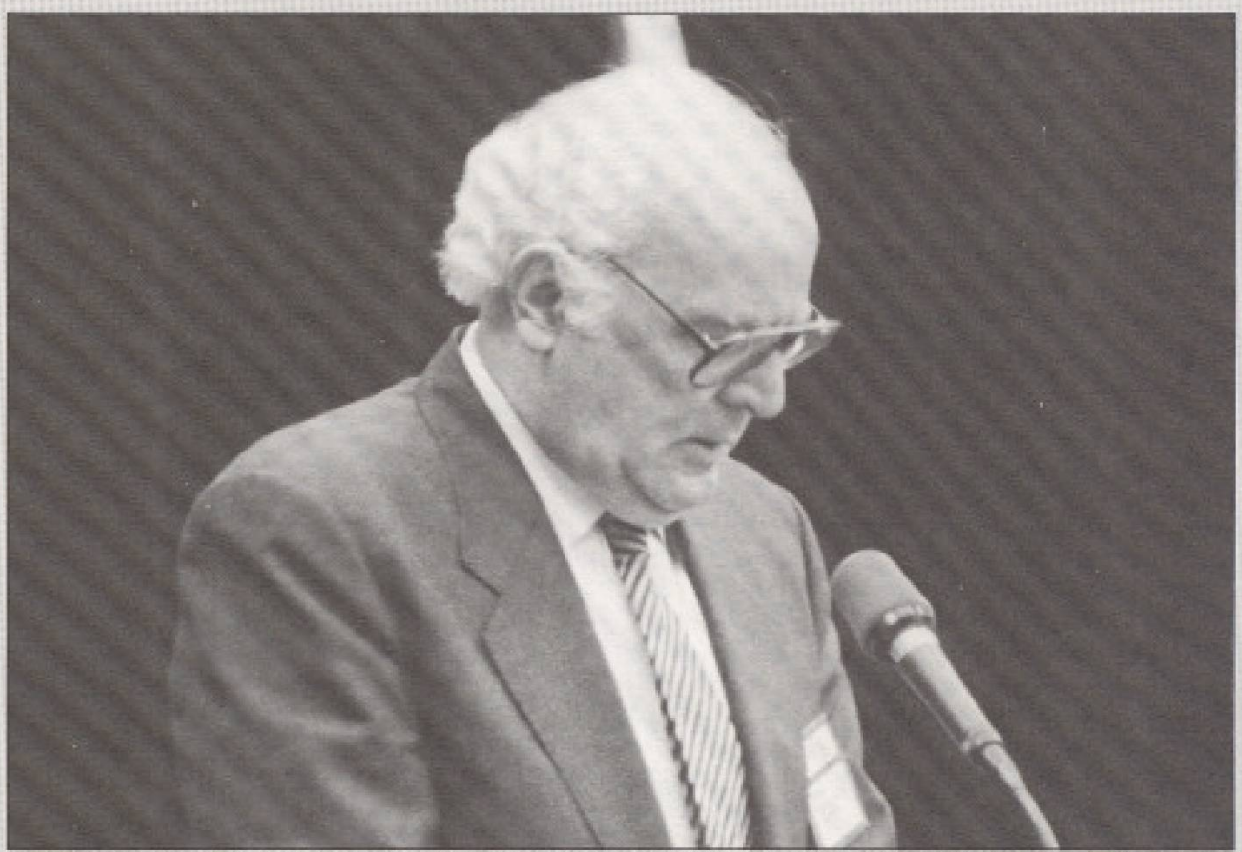

Monsieur Ántonio Enriquez Savignac, secrétaire général de l'Organisation mondiale du tourisme, introduit le thẻme du séminaire : tendance et défis du tourisme international.

tamment, d'un réseau mondial de Centres de formation en tourisme qui comprend, entre autres, déjà le Centre international de formation et de recherche en tourisme (CIFORT) de l'Université du Québec à Montréal; cl I'OMT entreprend aussi des actions pour protéger l'environnement et aider la planification et le développement durable; d) elle agitaussipour faciliter les déplacements de certains touristes (handicapés, etc.) et libéraliser la circulation des voyageurs; e) elle s'occupe d'aider les pays à faire leur promotion et leur marketing et finalement, IOOMT publie de nombreuses données périodiques très utiles pour la recherche et la prise de décision.

\section{L'OMT à Montréal}

En juillet 1992, I'OMT ouvrait a Montréal un Secrétariat régional des membres affiliés pour les Amériques. Cette réalisation fut une initiative de la Société du centre de conférences internationales de Montreal, de la Société du Palais des congrès de Montréal, du Centre d'études du tourisme et de l'UQAM; elle a reçu l'appui tangible des gouvernements du Québec et du Canada et de la Ville de Montréal.

En un an à peine, le Secrétariat régional de I'OMT à Montréal a déjá accompli beaucoup de choses. Il a dejà recruté une vingtaine de nouveaux membres affiliés quisontvenus s'ajouter aux quelques trente déjä existants dans les Amériques. On retrouve parmi ces nouveaux membres, Air $\mathrm{Ca}$ nada, Va Rail, Visa International, I'Institut de tourisme et d'hòtellerie du Québec. Texas A \& M University, Denver University, pour n'en nommer que quelques-uns. Acette activité de recrutement s'ajoute la diffusion gratuite d'un bulletin d'informations de huit pages composé d'articles traitant de sujets d'actualité ainsi que de bréves nouvelles. Ce bulletin, Viamericas, dont le premier numéro a été publié à la mi-avril, est rédigé en français, anglais et espagnol.

Enfin, le Secrétariat s'est engagé pleinement dans I"organisation du séminaire dont on trouvera dans les pages suivantes un premier compterendu lle Secrétariat publiera prochainement les Actes de ce séminaire). Mentionnons que cette rencontre a réuni plus de 200 participants dont plusieurs sont venus d'Amérique latine, d"Europe, etc. Dox-sept conférenciers ont traité de quatre grands thèmes dont les idées maitresses forment le contenu des pages qui suivent.

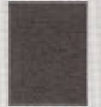

Première session: le tourisme à l'horizon 2000 et au-delà: perspectives mondiales et régionales

L'exposé de monsieur Enzo Paci, chef du département des statistiques de l'Organisation mondiale du tourisme, était divisé en deux parties: la première portait sur les variables exogènes et les forcesdu marché et la deuxième, 


\section{En collaboration avec :}

Gouvernement du Québec

Ministère du Tourisme

sur les previsions du tourisme mondial a l'horizon 2000 et au-dela.

A la suite de diverses études, l'OMT a identifie 6 variables exogènes pouvant influencer le tourisme: les changements démographiques et sociaux, la situation Economique et financière, les transformations politiques, législatives et réglementaires, les progrès technologiques, l'infrastructure des transports et la sécurité des voyageurs

Les forces du marché touristique sont les suivantes; a) la demande touristique sera marquée par une plus grande attention accordée aux préférences du consommateur; b) une augmentation des courts séjours, du tourisme d'awenture et des destinations a longue distance, c) de nouwelles demandes engendreront le développement de nouvelles destinations ainsi qu'une plus grande diversification du produit touristique dans les destinations bien établies; d) la compétitivité accrue obligera les acteurs touristiques a consacrer des budgets plus élevés aux activités promotionnelles; e) le secteur touristique privé sera témoin d'une concentration accrue du pouvoir.

En deuxième partic, monsicur Paci a présenté des prévisions statistiques relatives au phénomène touristique mondial. En voici les faits saillants.

- Lesarrivées de touristes mondiauxétaient de 25 millions en 1950 et de 456 millions en 1990. Elles atteindront 660 millions en l'an 2000.

- Les recettes du tourisme international (excluant le transport) sont passées de 18 milliards de dollars en 1970 à 255 milliards en 1990 ; elles atteindront $537 \mathrm{mil}$ liards en l'an 2000

- La région qui connaitra le plus fort taux moyen annuel de croissance pour les années 1990 a 2000 sera celle de l'Asie de l'Est et du Pacifique (avec 6,8\%).

- Les arrivées de touristes internationaux dans les Amériques devraient passer de 94 millions en 1990 à 147 millions en 2000.

- Toujours pour la région des Amériques, on remarque que la sous-région de I'Amérique du Nord connaîtra une diminution de sa part dans les arrivées de touristes d'ici l'an 2000 et ce, au profit de l'Amérique latine et des Caraibes.

- Au rang des principales destinations touristiques, on remarque que le Canada a subi une baisse considérable depuis 1950 , où il se classait au deuxième rang, pour atteindre le dixième rang en 1992 .

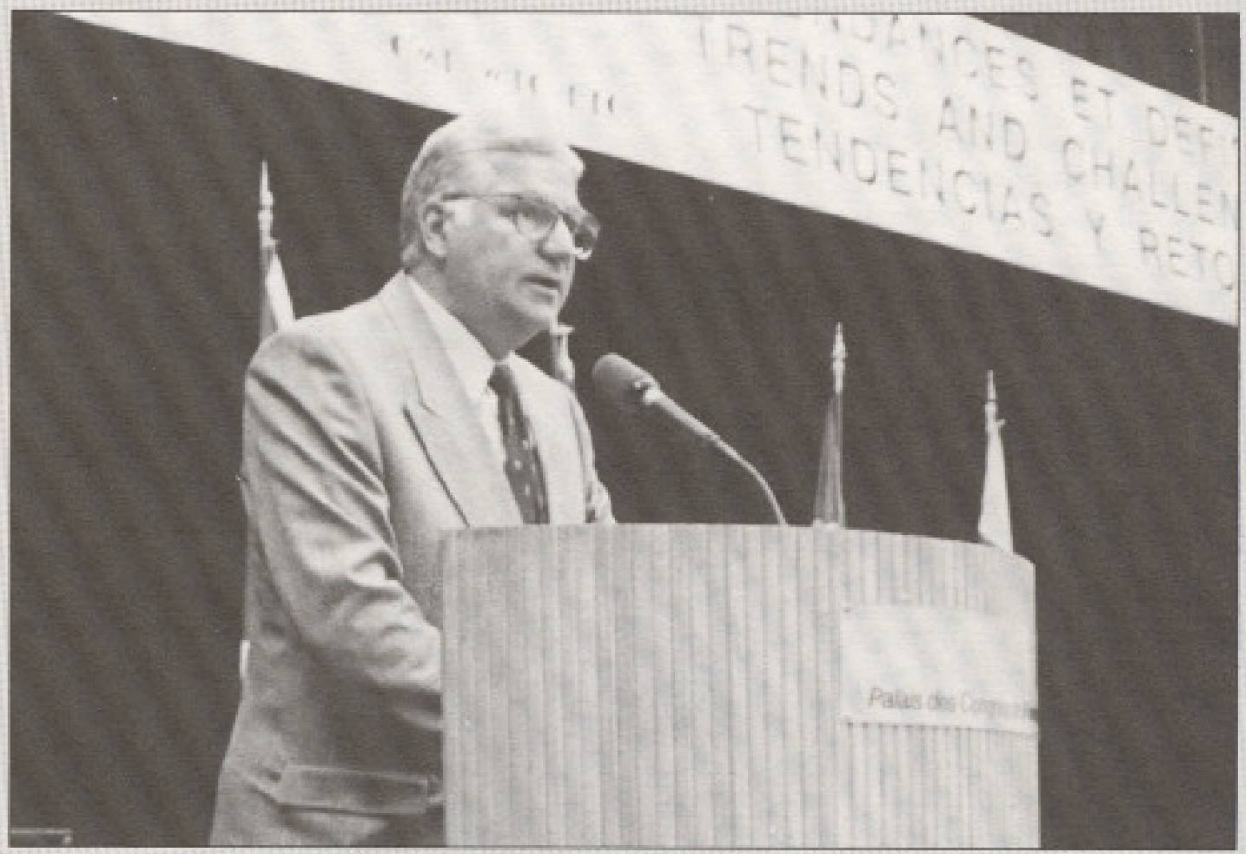

Monsieur Bruno Fragasso, président-directeur général du Palais des congrés et président du séminaire.

L'exposé de monsieur Michel Langlois, gouverneur de la Chaire de tourisme et directeur du module de gestion et intervention touristiques del "Université du Québec à Montréal a porté sur les enjeux mondiaux du marketing touristique: les défis du $\mathrm{XXI^{* }}$ siècle. Considérant differents aspects de l'evolution mondiale, tels que la surpopulation des pays du tier mondę, l'augmentation du travail à l'étranger, l'extension du chồmage, la déréglementation et la libéralisation des négociations commerciales et l'internationalisation des économies, une question se pose: $*$ Quiseront lesclients de l'industrie touristique au cours du XXI' siècle?

Dans un monde où l'économie de services prend de plus en plus de poids, la manière de faire des affaires ne sera plus ce qu'elle était. La compétitivité ne repose pas seulement sur les prix mais également sur l'innovation, la différenciation et la qualité.

Aussi, il y aura davantage de personnes qui auront le désir, le temps et les moyens de voyager. Mais qui contrôlera les flux monétaires engendrés par ces déplacements? Il faudra apprendre à gérer cette tendance lourde qui veut qu'il y ait de plus en plus de voyageurs aux attentes et aux besoins de plus en plus diversifiés et qui seront de plus en plus sollicités par des prestataires capables d'offrir mille et une propositions séduisantes aux allures magiques. Il faudra également composer avec une réalité incontournable et irréversible: l'industrie du tourisme et du voyage est, depuis 10 ans ou 20 ans, une des grandes aindustries du futur et l'une des chances économiques et sociales du siècle à venir. La concurrence sera féroce. II faut roconnaître que chaque coin de la planète peut prétendre avoir une expérience à proposer a des woyageurs. Comment alors influer ou composer avec le développement de ce fait majeur?
Les enjeux complémentaires au développement du secteur touristique seront relatifs à la croissance, a la structure, a la gestion de l'information, à l'économie et aux commerces.

Il ne faudrait pas oublier les risques auxquels doit faire face tout gestionnaire. Penser que les autres pensent comme nous, penser quece sont les vendeurs qui vendent et penser que nous ne souffrons pas de nombrilisme. Mais le piège majeur réside dans le fait de penser que les produits et les services se vendent de la mểme façon. Le voyage touristique est une expérience temporelle et spatialequi fait appelà une gamme d'émotions bien différentes de celles que l'on éprouvent quand on choisit de remplacer son téléviseur. Il faut donc cesser de vendre le voyage par le prix.

Le tourisme est une expérience. Jusqu'à maintenant, plusieurs entreprises ont considéré la valeur du produit touristique selon la qualité de son contenu. Cependant, dans les services, la qualité est une qualité perçue. Perçue par les clients. Ainsi, la survie d'une entreprise de services passe par la satisfaction de la clientèle. Une vente n'est pas le fruit du hasard. Il faut connaître les désirs et les attentes de sa clientèle. L'offre de services différente et intéressante doit l'être aux yeux de notre client et non baske sur nos propres expériences.

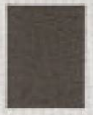

\section{Deuxième session: l'évolution des grands produits touristiques}

Le premier conférencier de cette session, , monsieur Howard Pickett de la firme Walt Disney, a traité des parcs de loisirs.

La compagnie Disney considère, en tout premier lieu, l'importance croissante du tourisme 


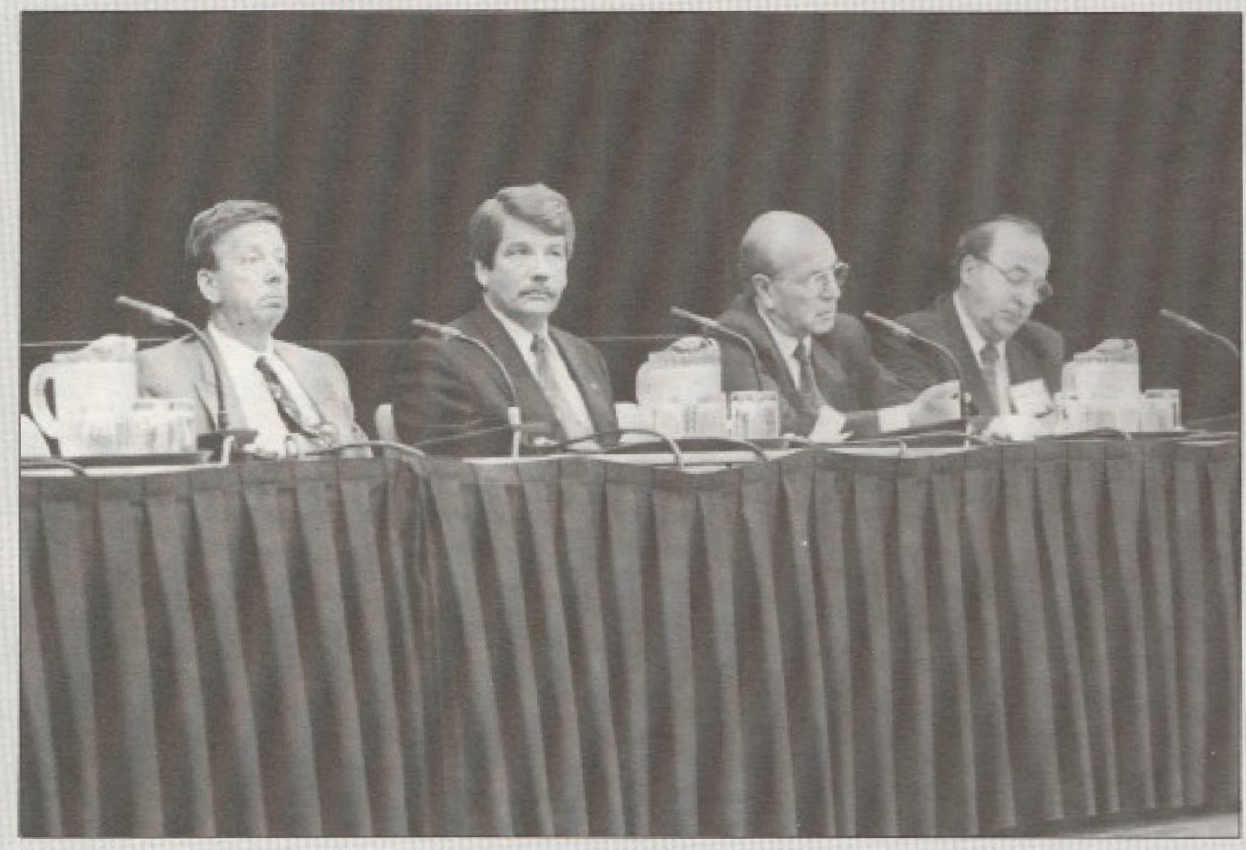

L'avenir du tounisme laisse-t-il songeur? Certaines personnalites de la súance d'ouverture s'interrogent sutrement aै ee propos. De gauche à droite : Monsieur André Vallerand, ministre du Tourisme du Québec, Monsieur Jean Doré, Maire de la Ville de Montréal. Monsieur Antoine Samuell, Directeur général du Tourisme, Industrie et Sciences du Canada, rógion de Québec et Monsieur Philippe Vaillancourt. Directeur du secrétariat régional des membres affiliés de l'OMiT pour les Amériques. Ont participés aussi a cette séance d'ouverture Monsieur Antoine Cachin, président du Comité directeur des membres affilies de l'OMT et Monsieur Bruno Fragasso, président-directeur général du Palais des congrès de Montréal et président du séminaire.

international. Elle mise avant tout sur le développement d'études de marché efficaces, lui penmettant de connaitre les attentes de ses différents types de clientèle.

Avec des agences de marketing basées dans divers pays du monde (notamment dans les villes de Londres, Toronto, Milan et Amsterdam), elle assure une visibilité accrue de ses services auprès des différents marchés mondiaux et de ses outils promotionnels innombrables. Que ce soit les nombreux films, les magasins spéxialisésoule Dinncy Chub, tousces moyens favorisent la visibilité de l'entreprise. Elle concentre présentement ses efforts sur l'analyse des marchés potentiels.

Le deuxième conférencier, monsieur Antoine Cachin, secrétaire général du Club Méditerranée et président du Comité directeur des membres affiliés de l'OMT. Il a analysé l'évolution des villages de vacances.

Les villages de vacances, image type de la formule de forfait tout inclus, se distinguent des autres produits touristiques par la facilité et la liberté daction des visiteurs, le rapport qualité/prix et l'intégration au site. Malgré les reproches que peuvent recevoir les villages de vacances (tels que leur fermeture sur l'extérieur), ils ont donné naissance à un nouveau concept de vacances et, petit à petit, ce concept a influencé une série de nouweaux produits de vacances.

Les diverses tendances du marché touristique, telles que la mondialisation et l'éclatement des marchés ainsi que la très forte montée de la

\section{En collaboration avec:}

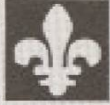

Gouvernement du Québec

Ministère

du Tourisme

Mais le principal élément est la suroffre à laquelle doit faire face l'industrie hôtelière. L'industrie doit également travailler avec des hôtels vétustes et en mauvaises conditions. Les perspectives d'avenir résident dans l'écoute du client. La reprise 6́conomique sera lente mais l'augmentation de la demande devraits'accélérer dú à cette portion grandissante des gens à la retraite dans la population mondiale.

On note cependant la présence de trois obstaclesmajeurs. La longue reprise 6 conomique, la faiblesse réelle de cette industrie et la compétition agressive sur les tarifs. Mais il y a également une opportunité qui s'offre à l'industric hôtelière. Le développement de systèmes de traitement de données et de distribution qui favorisera la connaissance des clients. L'arrivée de la THISCO (The Hotel Industry Switch Company) et la HCC (Hotel Clearing Corporation) qui travaillent au développement de nouvelles technologies, viennent leur assurer un avenir prometteur.

En fait, ce sont les chaînes d'hôtels qui, étant mieux organisées et mieux structurées, connaîtront une reprise plus rapide que les petites entreprises indépendantes. Les trois dernières années furent très difficiles pour l'industrie touristique en général mais grâce à des efforts bien dirigés, le secteur hôtelier parviendra à sumonter cette dure période.

Le quatrième conférencier, monsieur Julian Prins, directeur international des ventes pour Carnival Cruises, a discuté du marché des croisières.

L'industrie des croisières est un secteur qui offre un fort potentiel de développement sur le marche international. Cesegment favorisera le développement de nouvelles installations touristiques dans des régions encore peu connues.

Le troisième conférencier, monsieur Charles E. Brownfield, III, est vice-président au marketing etaux ventes pour les Amériques des Hôtels Inter-Continental. Il nous a parlé de l'évolution de l'hôtellerie.

Face a l'évolution rapide du secteur touristique, l'industrie hôtelière joue un rôle de plus en plus important. Aux Etats-Unis seulement, cette industrie procure 6 millions d'emplois. Pour bien préparer l'avenir, il est essentiel de regarder les expériences passées et d'analyser les facteurs qui auront un impact sur les développements futurs. Les principaux constats sont les suivants: diminution du tourisme d'affaires due à la récession et à la guerre du Golfe, augmentation des congrès et une augmentation du marché des loisirs
Au niveau de la demande, on prévoit que le nombre de passagers augmentera de 6 millions en 1990 à 8 millions en l'an 2000. La récession, qui tend à s'éterniser, amène des problèmestels que le manque d'investissements de la part des gouvernements et l'augmentation des coûts de construction des navires. Les prix des forfaits ont subi une baisse considérable, entraînant ainsi une certaine exigence de la part des clients face au rapport qualité/prix. Et ces clients recherchent de plus en plus des forfaits de courte durée. Face à ces contraintes et tendances, l'industrie des croisières devra s'ajuster en développant de nouvelles destinations et des nouveaux types de forfaits. L'internationalisation des croisières est un élément qui 


\section{En collaboration avec:}

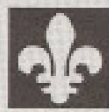

Gouvernement du Québec

Ministère

du Tourisme

devrait être favorable au développement et à la survie de ce secteur d'activité.

Le cinquième conférencier, monsieur Martin Brackenbury est directeur du Thompson Travel Group et président de la Fédération internationale des tours opérateurs.

Le secteur d'activité touristique que représente les tours opérateurs a connu un développement incomparable au cours des années 1960 où la demande était alors plus forte que l'offre. Mais au début des années 1970, les tours opérateurs ont dû affronter certains problèmes financiers. Et lesconsidérations environnementalesse font sentir. L'accès aérien devient de plus en plus difficile. Comment gérercepotentielquigénère des profits importants sans nuire à notre environnement? Cette question amčne les entreprises du secteur touristique ì reformuler leurs stratégies d'opération et de gestion.

Les tours opérateurs doivent également faire face à un autre problème: les gens recherchent de plus en plus des voyages non organises. Ils désirent planifier eux-mêmes leurs vacances. La recherche de liberté devient une tendance majeure. Il faut, une fois de plus, repenser nos stratégies de développement.

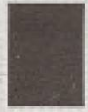

Troisième session: transport aćrien: impact des nouvelles tendances sur le tourisme

Madame Louise Robitaille, directrice des stratégies commerciales chez. Air Canada, a présenté le point de vue d'une société aérienne.

L'industrie aérienne a connu, et connaît toujours, d'énormes difficultés d'opérations. La crise économique a fait perdre, au Canada seulement, des milliers de dollars chaque jour. La situation se replace tranquillement. Mais d'autres problèmes surgissent. Les appareils sont vieux et en mauvais état. La congestion des aéroports devient une question stratégique. Il faut tenir compte de la protection de l'environnement dans nos opérations.

Cependant, la récession a également modifié les attentes des consommateurs. Il faut alors distinguer deux types de voyageurs: les clients d'affaires et les vacanciers. Lesclientsd'affaires recherchent d'abord et avant tout la qualité du service, la rapidité du transport. Le prix n'est pas le principal besoin a combler. Quant aux vacanciers, on remarque qu'ils considèrent le rapport qualité/prix comme un élément important dans leur prise de decision.

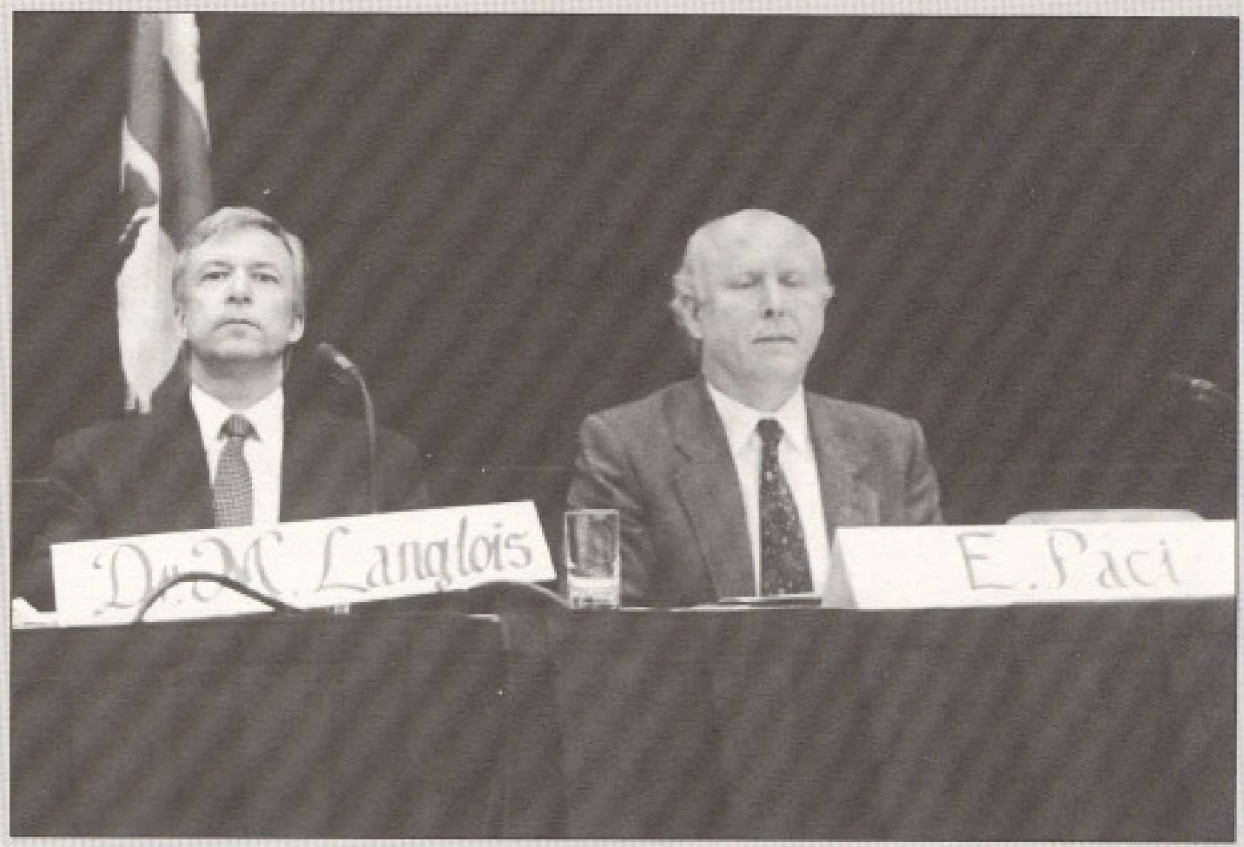

Messieurs Enzo Paci de l'OMT et Michel Langlois de l'UQAM se préparent à présententer deux des communications de début du séminaire qui ont situé d'emblée les exposés et les échanges à un trìs bon niveau.

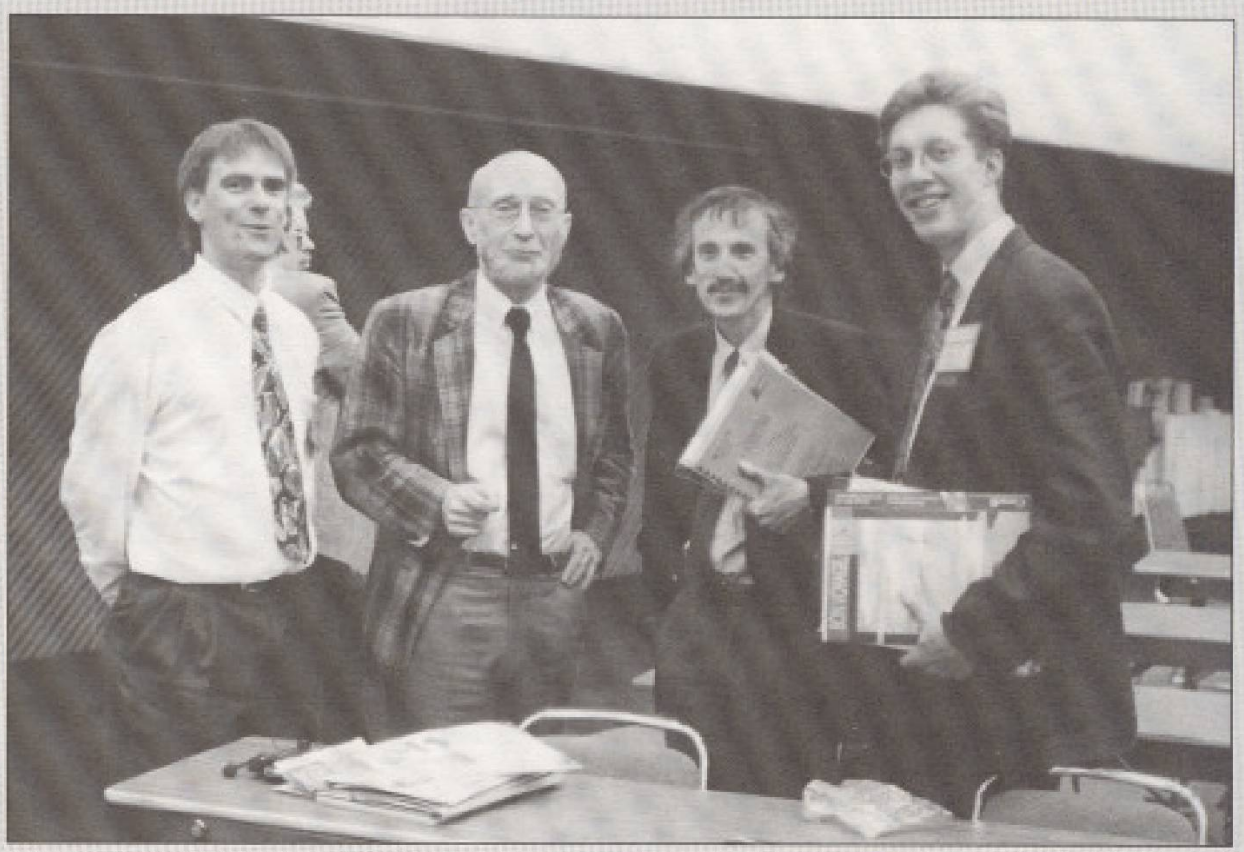

Messieurs Bernard Morrucci de la Sorbonne et Jean Stafford de I"UOAM, co-rédacteurs responsables du présent numéro de Téoros consacré à la prévision-prospective du tourisme, sont entourés ici de M. Charles Bélanger (á droite) dipiome en tourisme et presentement en rédaction de these de maitrise en sciences politiques a I'UOAM et do M. François Godin, étudiant au baccalauréat en gestion et intervention touristiques de I"UOAM. Le séminaire fut roccasion d'heureuses rencontres comme cellead

Face d ces changements de comportement des consommateurs et pour pallier aux différents problèmes financiers que connaissent les compagnies aériennes, madame Robitaille a proposé quelques solutions: il faut trouver des façons de réduire les frais d'exploitation sans nuire au service à la clientèle. La taille des entreprises est importante, il faut créer des alliances. Ces alliances stratégiques permettront un plus grand accès, une meilleure effica- cité. Elles créeront une synergie de pouvoir et développeront une certaine complémentarité. Il faut miser sur la qualité du service. $\mathrm{La}$ technologie est un élément important dont il faut tenir compte dans l'élaboration de nos stratégies de gestion.

Pourarriver à une reprise significative, l'industrie aérienne doit miser sur la consolidation. Sans quoi, elle court au suicide. 
Monsieur Paul M. Ruden, premier vice-président pour les affaires légales et l'industrie de l'American Society of TravelAgents (ASTA), a présenté le point de vue d'un utilisateur.

Il faut souligner d'abord que l'ASTA réunit 10, 000 agences de voyages américaines qui totalisent, à elles seules, 47,5 milliards de dollars de vente. Selon monsieur Ruden, les sociétés aériennes doivent tenir compte de certaines tendances et de certains comportements des voyageurs dans l'élaboration de leurs stratégies de gestion. La croissance rapide du nombre de passagers a amené une baisse de la qualité du service. Beaucoupde genssesentent inconfortables lorsqu'ils voyagent. Pour regagner la confiance des voyageurs, il faudrait assurer une qualité totale des voyages en avion. Pour arriver à surmonter cette période déficitaire, I'industrie aérienne doit miser sur la collaboration des autres secteurs touristiques.

Monsieur Chris Lyle est sous-directeur des études économiques et des statistiques pour l'Organisation de l'aviation civile internationale (OACI). Il nous a présenté le point de vue des organisations internationales.

Présentement, le secteur touristique et l'industrie aérienne utilisent différentes règles de jeu. L'aviation internationale est gérée par des accords bilatéraux complexes, souvent contrölés par les gouvernements, alors que le tourisme est peu réglementé, à moinsqu'il ne s'agisse des lois d'échanges commerciaux ou de concurrence.

Le défi majeur des années à venir sera d'augmenter les profits pour en arriver ensuite à les investir dans le renouvellement des équipements aériens. Ét lescompagnies aériennes qui forment cette industrie ont entrepris des actions qui s'orientent vers la collaboration et les ententes de marketing. L'arrivée des systèmes informatiques de réservation (CRS), a créé une guerre de pouvoir et d'accès. Les compagnies aériennes gèrent les systèmes de distributions internationaux (GDS) et les agents de voyages n'ont aucune part directe dans ces systèmes. Cette gestion contrôlée créera des problèmes de corruption. Comment le tourisme et le transport aérien peuvent-ils se rejoindre afin de former un tout favorable au développement de chacun? C'est d'abord grâce à l'implication d'organisations telles que I'OACI et l'OMT que nous atteindrons les buts fixés.

Monsieur Kevin Dobby, directeur régional de l'Association du transport aérien international (IATA), a présenté un deuxième point de vue concernant l'impact des nouvelles tendances du transport aérien sur le tourisme.

En premier lieu, il a fait état de la situation actuelle de l'industrie aérienne. Le transport aérien est en crise. La situation financière est désastreuse. 200 compagnies aériennes ont subi des pertes en 1991. En 1992, les choses se sont empirées: malgré une augmentation de 13 \% du trafic aérien, les compagnies offrant des vols réguliers ont subi des pertes combinées de plus de 4,8 milliards de dollars.

Mais comment peut-on expliquer ce revirement, si l'on compare la situation actuelle aux années 1980 où la situation était alors près de lexcellence? L'élément principal vient de la surcapacité du marché De 1990 à 1992, la capacité a augmenté de $23 \%$ alors que la demande n'augmentait que de $14 \%$. Les compagnies aériennes ont tenté alors de réduire leurs coûts d'opération. On effectue de nombreuses mises à pied, on fait appel z̀ des consultants. Des pressions sont faites auprès des compagnies aériennes pour qu'elles réajustent leur structure de prix.

Mais il existe un élément majeur qui viendra transformer cette industrie au cours des prochaines années: la privatisation des compagnies aériennes. Cette privatisation, en plus de provoquer la chute du pouvoir de l'Éat, créera de nombreuses opportunités de marketing. Comment l'industrie touristique doit-elle réagir face à cette situation plutôt négative? Selon monsieur Dobby, la capacité des aéroports est un elément assez embarrassant. II faut trouver des solutions qui viendront réduire cette pollution. Les taves imposées par les gouvernements sont bien loin d'offrir des solutions parfaites,

La solution idéale réside plutôt dans la création de liens entre les differentes industries qui forment lesceteur touristique. Il faut combiner les capacités du transport aérien aux autres secteurs.

En tant que representant d'une organisation mondiale, monsieur Enzo Paci, chef du département des statistiques de l'OMT, est venu exposer les résultats d'études récemment effectuces.

L'une de ces études, commandée par le Secrétariat général de l'OMT, porte sur les politiques du tourisme et de l'aviation, avantages et inconvénients. Cette étude, qui présente à la fois des résultats globaux et des résultats distincts, par pays, procurera un cadre d'analyse à l'industrie. Les conclusions générales de cette étude sont les suivantes: l'aviation et le tourisme ont beaucoup de souplesse. Ils ont crû plus que le $\mathrm{PNB}$ au cours des 20 dernières années. Le transport aérien a engendré de nouvelles sources de touristes. La protection des compagnies aériennes entre en conflit avec l'expansion et l'internationalisation de ces compagnies, affectant ainsi l'expansion de l'économie. L'industrie touristique génère de nombreux emplois, beaucoup plus que certains autres secteurs de l'économie qui eux, obtiennent les faveurs des gouvernements.

Voici maintenant les conclusions amenées par les constatations précédentes, qui viennent directement affecter le secteur touristique.

- Malgré lespressions faites parl'OMT, les études sur les politiques ne sont pas suf-
En collaboration avec:

Gouvernement du Québec

Ministère

du Tourisme

fisantes. Il est capital de mieux connaitre l'apport de ce secteur' dans l'économie.

- Le tourisme n'est pas encore considéré comme une importante industrie dans les politiques gouvernementales. C'est grâce aux études ci-haut mentionnées que nous réussirons à prouver le poids de cette industric.

- Il faut accorder une plus grande priorité au tourisme (un seul ministre chargé du tourisme, parfois même aucun. Le tourisme est intégré dans un autre Ministère).

- Il faut s'assurer de l'existence d'une association pour défendre les intérêts du tourisme auprès du gouvernement.

- Lespaysdevraienteux-mêmesétablirleurs propresstatistiques relatives au tourisme.

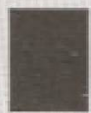

Quatrième session: les systèmes
d'information dans la distribution
des produits touristiques

Le premierconférencier decette dernière session, monsieur Alan Stark, présidentdirecteur général d'Amex Canada Inc., affirme que les systèmes informatiques de réservation (CRS) représentent un enjeu très important pour le développement de l'économie touristique mondiale. Ces systèmes sont encore au stade de l'enfance et l'avenir semble être prometteur.

Les CRS ont complètement modifié la façon de gérer le service à la clientèle. Que sera l'avenir des systèmes informatiques de réservations? Premièrement, il y a la mondialisation. Puis, il y a la consolidation.

Monsieur Georges Vialle, directeur de l'informatique au Club Méditerranée, a présenté les différents éléments qui gèrent la distribution informatique de produits touristiques.

Pour mieux comprendre et prévoir l'avenir, il faut regarder l'évolution du secteur. Grấce aux nouvelles technologies, nous sommes en mesure de trouver des solutions pour répondre aux nouveaux enjeux qui se présentent au secteur touristique. Par l'utilisation de systèmes desplus efficaces, le ClubMed offre un systìme de référence pour le secteur. Il couvre l'ensemble de la filière, il est conçu pour répondre aux besoins commerciaux de l'ensemble des pays du monde, il traite de l'ensemble des produits, il peut héberger plusieurs sociétés, il estuniversel (selon une ćtude réalisće en 1992) et finalement, le Club est ouvert au partenariat.

Le troisième conférencier, monsieur Frank Camacho, est vice-président de la Corpora- 
En collaboration avec :

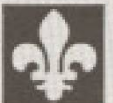

Gouvernement du Québec

Ministère

du Tourisme

tion ITT Sheraton, directeur du marketing, division nord-américaine.

Pour l'industrie hôtelière, tout comme pour l'ensemble de l'industrie touristique, l'électronique permet dedonner le service rapide auquel le client s'attend. Les sociétés hôtelières ont mis de l'avant des systèmes de réservation très complexes. Ils ont travaillé au développement de leur propre système. Cependant, ils ont oublié que ces systèmes doivent être interreliés pour être efficaces.

Pourquoi alors ne pas utiliser les systèmes deja mis en place par les compagnies aériennes? Les systèmes aériens ne comprennent pas les données du secteur hôtelier. Les différents produits qu'offre le réseau hôtelier sont difficiles à distribuer par le réseau aérien.

En conclusion, l'auteur a souhaité voir des systèmes de réservation beaucoup plussimples. Il lance un défi aux acteurs du milicu touristique: Travaillons de façon plus efficace et choisissons une langue de communication plus facile. Nous devons travailler ensemble.

Aux questions posées par des utilisateurs, des opérateurs de systemes informatiques de distribution ont cherché à donner des réponses.

Monsieur Bernard Anquez est directeur général duplanning stratégique pour AMADEUS. Ce systeme de distribution dessert actuellement plus de 320 lignes aériennes. Plus de 81 compagnies sont distribuées par Direct Access et 77 par AMADEUS Access, qui représente un niveau de distribution très sophistique. *AMADEUS is The only GDS in 'The world that offers The technology of AMADEUS Access: it consist of a "Host to Host" data connection between The AMADEUS Data Centre and The inventory of "The airline.

AMADEUS présente les horaires et les disponibilités des vols sur un affichage neutre, en temps réel. Il offre également aux compagnies aériennes une distribution equitable.

On peut done affirmer que l'industrie des GDS inclut un joueur européen, AMADEUS. Mais que lui reserve l'avenir? En 1993, une entente de coopération avec les GDS américains et ssiatiques devrait être conclue. Il tente également de finaliser une entente avec les compagnies ferroviaires SNCF et Deutsche Bundesbahn. Et finalement, il va continuer à intéresser de nouveaux fournisseurs tels que des tours opérateurs, des traversiers et d'autres produits touristiques.

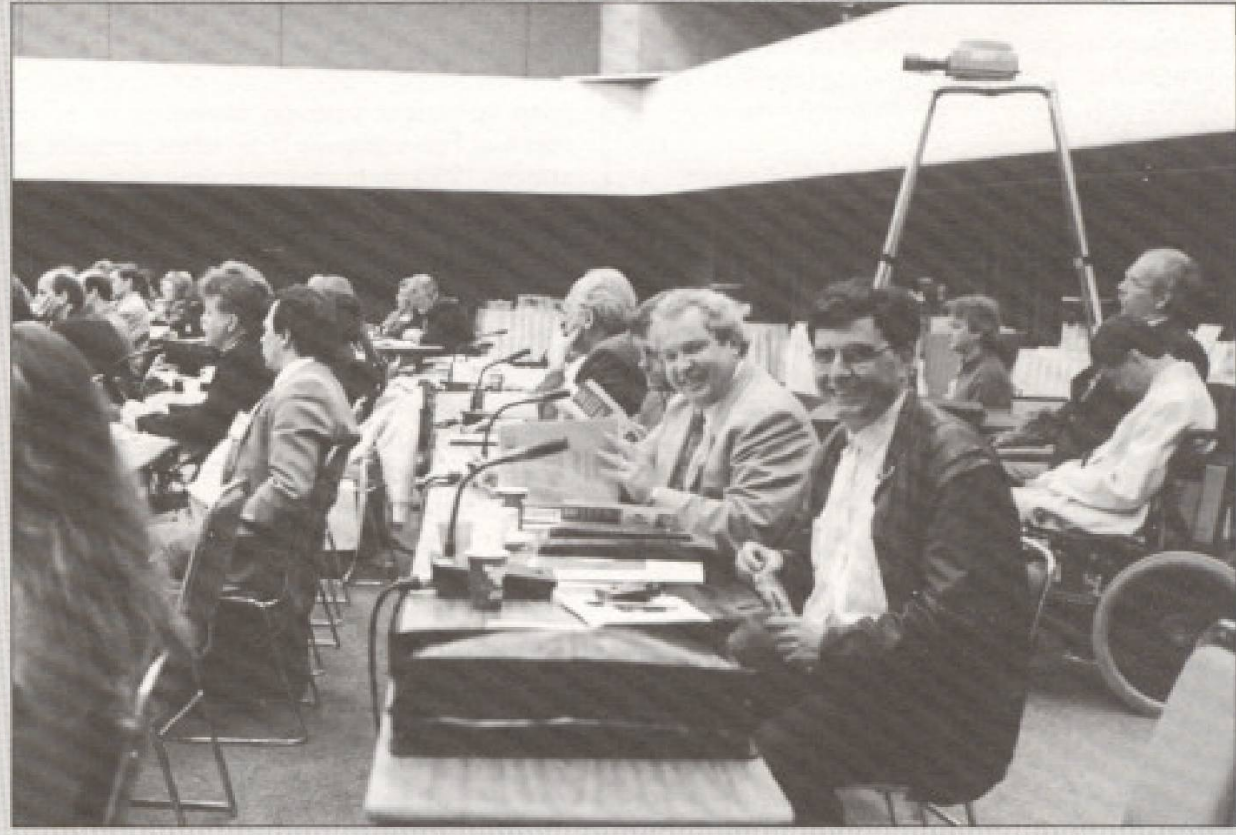

Dans l'ensemble, les participants ont sumi avec beaucoup d'attention les présentations des contérenciers. Toutefois certains n'ont pas ignoré la présence du photographe dans la salle.

Pour conclure, il est important de mentionner que les GDS doivent être considérés comme des membres de l'industrie touristique, et non seulement comme un club oligopolistique qui ne fait que profiter de ses fournisseurs!

Monsieur David Collier est vice-président pour l'Europe de SABRE Travel Information Network (STIN).

A l'heure actuelle, l'industrie des GDS secompose de troissystèmes soit SABRE, GALILEO et AMADEUS. «SABRE possède déjà le leadership de cette industrie. En 1993, nous prévoyons effectuer plus de 250 millions de réservations. Nous croyons que pour être prospère, il faut penser mondialement et agir tocalement.:

Le principal rôle des CRS est de créer un cadre de réseau de distribution pour l'industrie touristique en entier. ${ }^{\circ C R S}$ are key to The distribution of Travel products. Ourgoal mnst be to link travelers with Travel products by building a bridge through information technology and therefore make it easier for The consumer to purchase Travel products in preference to his HI FI or car.*

Monsieur Didier Delepine, directeur général adjoint pour la Société internationale des télécommunications aéronautiques (SITA), a présenté sa vision quant aux systèmes de distribution.

La déréglementation de l'industrie aérienne et des télécommunications ainsi que l'évolution rapide des nouvelles technologies viennent directement affecter la façon de gérer le secteur touristique. Nous devons alors développer des systèmes d'information efficaces et efficients.
Actuellement, il existe plusieurs systèmes de distribution qui furent développés par differentes entreprises. Le rôle de la SITA est de garder ensemble ces systèmes, d'assembler les pièces, de favoriser la coopération. L'industrie touristique doit miser sur la transparence, l'accès rapide aux différents systèmes et la mondialisation. La qualité réside dans la capacité de fournir aux consommateurs des solutions efficaces.

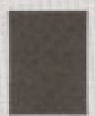

\section{Quelques grandes} conclusions du séminaire

Un thème aussivaste que Tendances et défis du tourisme international, traité par 17 spécialistes en deux jours, faisant appel essentiellement aux grands intervenants de l'industrie puisque ce séminaire était une initiative du Comité des membres affiliés de l'OMT, ne se prête pas facilement à l'exercice de synthèse. Quand paraîtront les Actes de ce séminaire, quand chacun pourra, a loisir, décrypter les messages de chaque communication; les lignes de force deviendront peut-être plus évidentes.

Pour terminer ce compte rendu, il nous a semblé que l'intervention finale de monsieur Antoine Cachin, président du Comité des membres affilies, pourrait trìs bien servir a faire un bilan provisoire de ce séminaire.

Comme le veut généralement l'analyse touristique, ce bilan traite d'abord séparément de la demande et de l'offre touristique.

Dans l'évolution observable de la demande, l'auteur a retenu deux tendances de fond, soulignées par plusieurs conférenciers: Ia mondialisation desmarchés et, en même temps, 


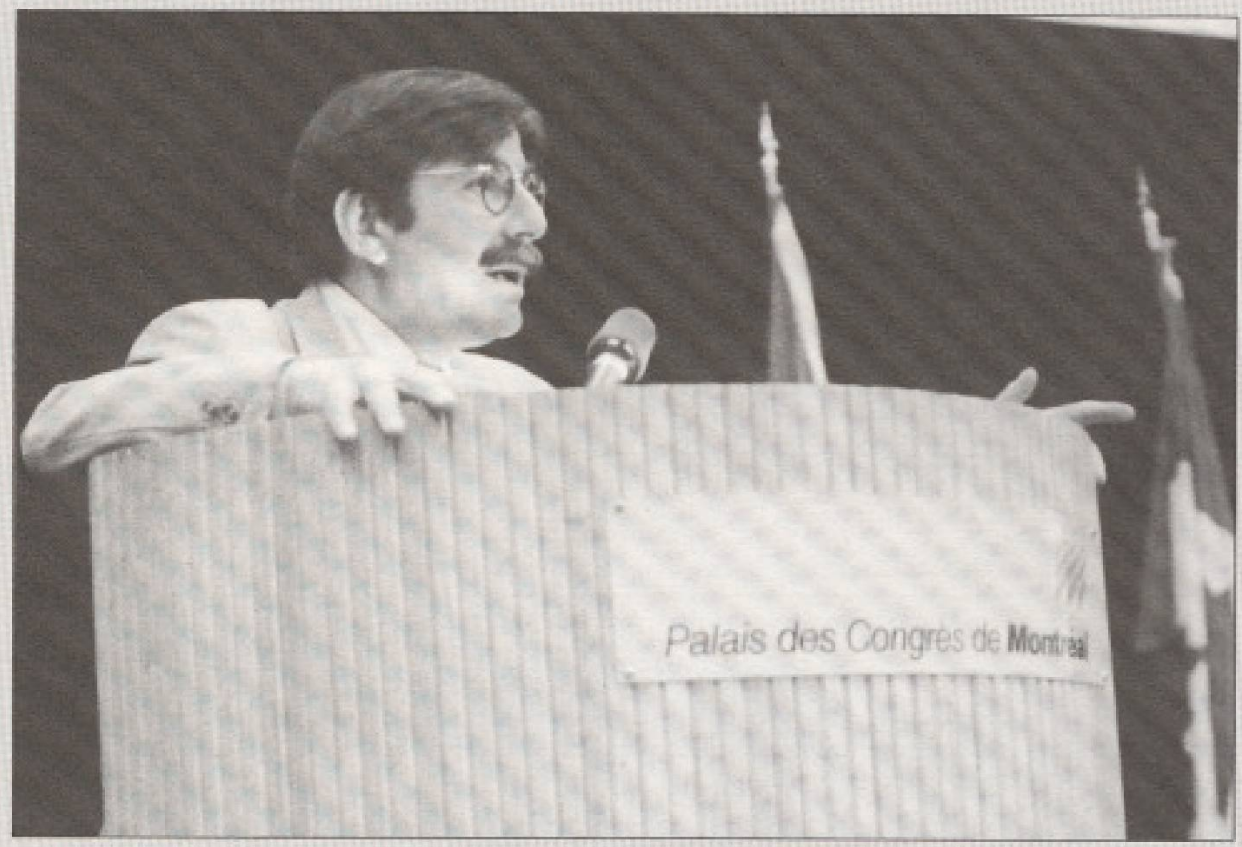

Monsieur Antoine Cachin, président du Comité directeur des membres affilés de l'OMT et secrétaire géneral du Club Méditerranée au moment de faire le bilan du séminaire

leur éclatement. L'opposition entre ces deux tendancesest plusapparenteque réclle, comme on le voit en s'attardant à chacune d'elles.

Que veut dire mondialisation des marcbés? Cette expression réfere à des tendances dans les demandes des consommateurs actuels et potentiels, tendances qui tentent à se généraliser à de très nombreuses et diverses clientèles dans les pays industrialisés. A. Cachin a traité de quatre de ces tendances lourdes de la demande touristique internationale, sans prétendre épuiser le sujet:-

a) tendance à demander des produits *parfaits*: services parfaits, prestations parfaites, dans des environnements parfaits. La demande de qualité semble irréversible: les consommateurs sont, de plus en plus, des connaisseurs sachant ce qu'ils veulent. Ceci sera un des grands défis pour l'offre de demain;

b) tendance à reconsidérer énormément le facteur prix dans le processus d'appréciation des produits et dans les décisions de consommer. En homme d'action, monsieur Antoine Cachin relève aussitôt qu'il n'est passimple depenser, en même temps, haute qualité et bons prix, surtout en temps de récession...;

c) tendance à rechercher, nouveau, des valeurs sûres, de l'authentique, après le temps du clinquantet du gadget; c'est une autre dimension de la recherche de qualité;

d) tendance, enfin, vers des activités moins thard\%, plus sobres: le golf plutôt que le tennis; demême, demande plus forte pour

\section{En collaboration avec :}

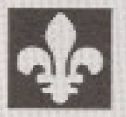

Gouvernement du Québec

Ministère

du Tourisme

treprises est nécessaire parce que les investisse ments a faire dans ce secteur sont de plus en plus lourds; il faut travailler a la grandeur de la planète pour dominer le problème de la saisonnalité; il faut se diversifier, ne pas mettre tous ses oeufs dans le mêtme panier, a cause des incertitudes monétaires, politiques, etc.

Ces arguments en faveur de la concentration internationale et des stratégies de production au niveau mondial ne sont pas tous propres au tourisme. Mais les raisons expliquant l'autre grande tendance de l'offre touristique: la forte montée de la concurrence, sont plus propres a cette industrié: il y a beaucoup d'entrants dans ce secteur et souvent, chacun croit pouvoir réussir seul; les états ont dérégulé beaucoup en tourisme; enfin, la récession a créé des surcapacités coûteuses dans cette branche économique.

Monsieur Cachin n'a pas semblé trop inquiet de ces grandes tendances du côté de l'offre comme de la demande touristique parce qu'il réagit en entrepreneur et voit aussitôt ces evolutions probables comme des défis à relever pour l'avenir à court ou à moyen terme.

Lesdefisetcommentles relever. La mondialisation exigera deux démarches très différentes: d'une part, creer des produits intemationaur, avec des personnels internationaux et des produits capables de satisfaire des attentes et des goûts largement partagés à travers le monde qui voyage; parallèlement, créer des produits nutionaux tris typés pour attirer des clientèles nouvelles (ex.: un Club Med italien pour des Italiens: Valtour).

Face à la segmentation des marchés, l'auteur comme plusieurs autres - s'engage à plein régime dans des recherches de flexibilité et de diversification. Il faut arriver à servir en même temps et dans un même lieu plusicurs groupes ayant des intérêts forts différents; mais il importe aussi de savoir rapidement changer de style pour servir successivement des clientèles différentes; enfin, le mot-clé: diversification, est le nouveau lcitmotiv: produire spécialcment pour chaque clientèle, varier les sites (pas très facile maintenant), trouver l'originalité face aux concurrents, etc.

II faut souligner en terminant, que monsicur Cachin, après quelques autres, a affirméqueces défis ne seront pas bien relevés sans un formidable effort du côté des ressources humaines: l'idéal sera d'avoir des travailleurs totalement internationaux (multiculturels) et totalement flexibles. Ces conclusions nous ont touchés en plein coeur! 

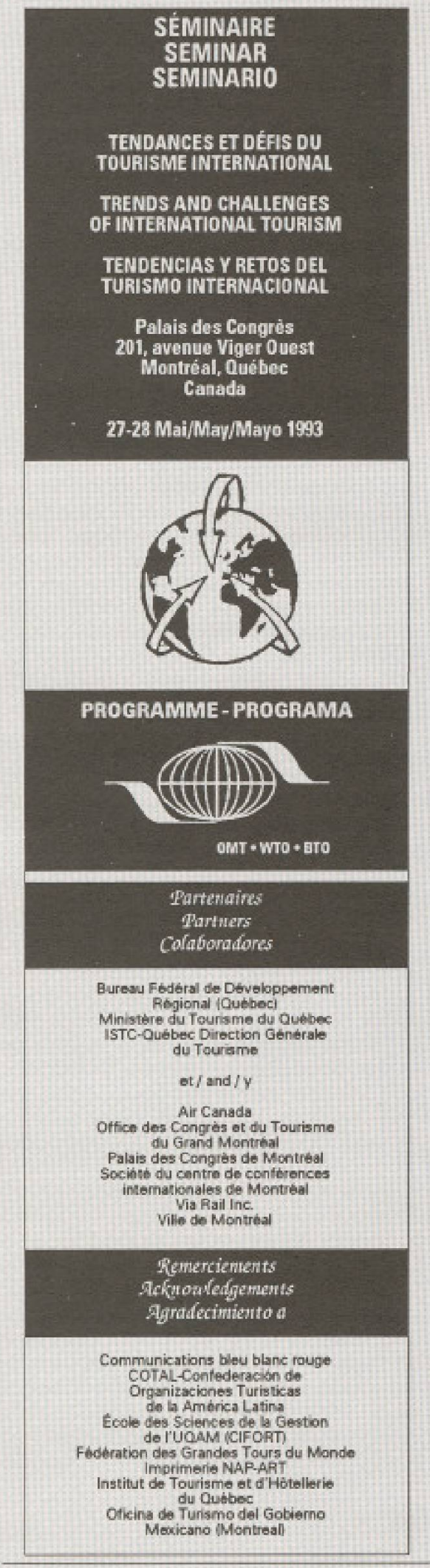

En collaboration avec:

Gouvernement du Québec

Ministère

du Tourisme
Horaire du stminalre

\section{LE MERCREDI 26 MAI}

13h00 Inscription dans le Hall d'acoueil, niveau principal.

I7hoo Reception de bienvenue offerte par la Ville de Montréal, a IHótel de Ville, au 275, rue Notre-Dame est

\section{LE JEUDI 27 MAI}

8h30 Inscription

9 hoo Seance d'ouverture

M. Bruno Fragasso, président-directeur génóral du Palais des congres et président du séminaire

M. Antonio Enriquasz Savignac, Secrétaire général de l'Organisation mondiale du tourisme

M. Jean Dort. Maire de la Ville dé Montréal

M. Andro Vallerand, Ministre du Tourisme du Québec

M. Antoline Samudili, Directeur général du tourisme, Industries et Sciences, Canada, région de Québec

\section{Premidre tastion}

Touriame a Thorizen 2000 et au-delk: perspectives mondiales et réglonales

$9+30 \quad$ Perspectives de croissance mondiale et régionale di l'horizon 2000

M. Enzo Pael, Chef du departement des statistiques, Organisation mondiale du tourisme

$10 h 15 \quad$ Pause-afte

IOh30 Les enieux mondiaux du marketing touristique: les defis du XXI" sidele

M. Michel Langlois, Gouverneur de la Chairede tourisme et de recherche en tourisme (CIFORT), Centre intemational de formation et de, Ecole des sciences de la gestion de l'Université du Oúbbec a Montréal (UOAM)

11 h45 Debat

12 h30 Déjeuner-Offert par la Société du Palais des congrès de Montréal,

\section{Dauxidima testan}

L'bvolution des grands produhts tourlatiques

14 hoo Pares de loisirs

$14 h 20$ M. Howard Pickett, Viceprésident, marketing. parcs Walt Disney Company

Villages de vacances
M. Antoine Cachin, Secrétaire gúnétal, Cub Mediterranée et président. Comité directeur des membres affiliés, Organisation mondiale du tourisme

14 h40 Hotellerie

M. Charles E. Brownfield, II, Vice-président, marketing et wentes, Amériques, Inter-Continental Hotels

15 hoo Pause-café

15 hio Croisières

16hoo Tours opérateurs

M. Martin Brackenbury. Directeur, Thomson Travel Group et président, Fédération internationale des tours operateurs

$16 h 30$ Debat

\section{LE VENDREDI $28 \mathrm{MAI}$}

Troisibme session

Transport abrien: Impaet des nouvelles tendances sur le tourlame

9hoo Point de vue d'une société aérienne

$9 h 30$ Mme Louise Robitaille, Directrice, stratégies commerciales. Air Canada

$9 h 30$ Point de vue d'un utilisateur

M. Paul M. Ruden, Premier vice pressident, Affaires légales et Industrie, American Soctety of Travel Agents (ASTA)

10hoo Pause-cafe

10 15 Point de vue des organisations internationales

M. Chris Lyle, Sous-directeur des études fconomiques et des, Organisation de laviation civile internationale $(\mathrm{OACl})$

M, Kovln Dobby. Directeur régional, Asscciation du transport aérien international(IATA)

11 h45 Debat

12 h30 Déjeuner-Offert par la Société du centre de conférences internationales de Montréal,

\section{Quatribma andion}

Lea systemes d'information dans le distribution des produfts touriatiques

14 hoo Les besoins des utilisateurs

M. Alan Stark, président-directeur général, Amex Canada Ine.

M. Georgen Vialle, Directeur, informatique, Club Méditerrande

M. Frank Camacho, Vice-président, Corporation ITT Sheraton, Directeur du marketing, division nord. américaine

$15 \mathrm{~h} 30 \quad$ Pause carté

$15 h 45$ La réponse des opérateurs

M. Ber nard Anquaz, Directeur général du planning stratécique, AMADEUS

M. David Collier, Vice-président, Europe, SABAE Travel Information Network ISTIN)

M. Didier Dolepine. Directeur gúnéral adjoint, Société internationale des telbcommunications aéronautiques [SITA]

$17 \mathrm{~h} 15$ Debat

1 hoo Cloture

M. Antonio Enriquez Savignac, Secrétaire général, OMT

M. Antoine Cachin, Président, Comité directeur des membres atfiliés, OMT 